\title{
Prognostic Markers of Symptomatic Congenital Cytomegalovirus Infection
}

\author{
Roberta Maia de Castro Romanelli ${ }^{1}$, Jean François Magny ${ }^{2}$ and François Jacquemard ${ }^{2}$ \\ ${ }^{I}$ Clinical Hospital, Federal Univerisity of Minas Gerais, Belo Horizonte, MG, Brazil; ${ }^{2}$ Institut de Puericulture et Perinatologie, Paris, France
}

\begin{abstract}
The objective of this research was to identify maternal and fetal characteristics as prognostic markers of congenital cytomegalovirus (CMV) infection. This is a descriptive study of 13 cases of congenital CMV infection referred to Institute de Puericulture et Perinatologie de Paris (IPP) from January 2005 to October 2006. Amniotic fluid puncture was performed to research CMV polimerase chain reaction (PCR). Cordocentesis and cord blood samples at delivery were also analyzed to determinate fetal platelets count, GGT, ASAT, ALAT, CMV-DNA and IgM antibody. Variables of symptomatic and asymptomatic infants were then compared. Data were analyzed by SPSS - 15.0. Mean gestational age of amniocentesis was $\mathbf{2 4 . 6}$ weeks and there was no difference of mean viral load in amniotic fluid considering infant features. Mean gestational age of cordocentesis was 26.1 weeks. There were no statistical differences of fetal viral load, IgM, platelets, GGT, ASAT and ALAT analyzed at cordocentesis samples, but at delivery, mean values of IgM and ASAT of fetal blood were increased in symptomatic ones ( $p=0.03$ for both parameters). When considering groups with normal and abnormal parameters, ASAT of cordon samples was also increased in symptomatic infants $(p=0.02)$. Sensibility, specificity, positive and negative predictive value of fetal ultrasound anomalies to detect symptomatic infants were, respectively, $80 \%, 62.5 \%, 57.1 \%$ and $83.3 \%$. Thus, identification of markers of CMV symptomatic infants should be aimed. Prenatal diagnosis, identification and follow up of congenital CMV infected infants are important to consider treatment for symptomatic infants, trying to avoid or reducing some possible sequels.
\end{abstract}

Key-Words: Cytomegalovirus, disease transmission, vertical, prenatal diagnosis, prognosis.

Cytomegalovirus (CMV) is the most frequent cause of congenital infection, affecting $0.2-2.2 \%$ of all live births in the United States [1]. In France, the rate of congenital infection is also in this range, approximately $1 \%$ [2]. In Brazil, studies showed prevalence from $2.1 \%$ [3] to as high as $6.2 \%$ [4]. Generally, $10 \%$ of infected children are symptomatic, while 85 $90 \%$ are asymptomatic. Among symptomatic infants, until 30\% of mortality are reported and most survivors (90\%) will have severe neurological sequels. Among the asymptomatic infants, 5 to $10 \%$ can develop sequels[1,5].

Although intrauterine infection may be the consequence of either a primary or recurrent infection, rates of vertical transmission are higher in the first episode: about 30-57\%, comparing to 0.1 to $3 \%$ in recurrences [1,5]. However, severely affected infants and adverse outcomes are more likely when infection occurs in the first half of pregnancy of primary infections [1,5]. According to the series of Liesnard et al. [6], severe disease is more common when mother infection occurred before 20 weeks of pregnancy. In a study conducted by Enders et al. [7] about prenatal diagnosis and outcome of infants with congenital CMV infection, authors concluded that fetal infections are more common when maternal infection occurs later in pregnancy but severity of fetal involvement is higher before 18 weeks of pregnancy.

Studies about other prognostic factors to identify infected fetus with greater probability of severe disabilities are being Received on 15 September 2007; revised 11 January 2008.

Address for correspondence: Dr. Roberta Maia de Castro Romanelli. Av Alfredo Balena 190, Hospital das Clinicas - 1st floor (Neonatology). Zip code: 30130-100. Belo Horizonte, MG, Brazil. Phone: (31) 34099383. E-mail: rmcromanelli@medicina.ufmg.br.

The Brazilian Journal of Infectious Diseases 2008;12(1):38-43. (C) 2008 by The Brazilian Journal of Infectious Diseases and Contexto Publishing. All rights reserved. conducted. Ultrasound anomalies, viral load in amniotic fluid and fetus samples are same examples of discussed topics [6,813]. But these parameters are not well defined. The present study aimed to identify maternal and fetal characteristics as prognostic markers of symptoms of congenital cytomegalovirus infection in a series of cases.

\section{Material and Methods General Concerns}

This is a descriptive study of 13 cases of congenital CMV infections that were diagnosed at Institute de Puericulture et Perinatologie de Paris (IPP) from January 2005 to October 2006. Infected pregnant women were referred to IPP for prenatal diagnosis according to seroconversion identified at basic level of care assistance. Maternal seroconversion was defined as: - appearance or presence of IgM anti-CMV, - appearance or increase of anti-CMV IgG, - low titters of IgG avidity, according to laboratory references.

During routine follow up, ultrasound evaluations were performed every four weeks after serological diagnosis. If fetal infection was confirmed (by positive polimerase chain reaction of CMV in amniocentesis samples), image exams were performed every two weeks. Images by magnetic resonance (IMR) were performed once after 32 weeks of pregnancy.

Amniocentesis was performed in all cases after maternal negative viremia and after 19 weeks of gestation, at least six weeks after seroconversion. Twenty mililitres of amniotic fluid were collected by abdominal puncture under continuous ultrasound guidance. Viral fetal infection was defined by presence of viral genome in amniotic fluid, also called as viral load (PCR performed by enzymatic activation/Lightcycle $\left.{ }^{\circledR}\right)$. Although amniocentesis was repeated in some cases, only the first one was considered for analysis. 
Cordocentesis was performed after positive results of CMVPCR in amniotic fluid. Fetal blood was used for determination of hematological (platelets count) and biochemical parameters $(\gamma$-glutamyl transferase-GGT, aspartate aminotransferase-ASAT and alanine aminotransferaseALAT), but also for detection of CMV-PCR and CMV specific IgM antibody. Although this procedure was performed twice in five cases, only first samples were considered for analysis. Cordon samples at birth were also obtained to perform all these tests (platelets, GGT, ASAT, ALAT, viral load and IgM). Postnatal data also included clinical evaluation and transfontanelle ultrasound.

Platelets count was performed by Abbott Cell-Dyn ${ }^{\circledR}$ (reference value: 150,000 to 400,000 g/dL). GGT, ASAT and ALAT were performed by Konelab 30 - Kinetic: $37 \AA$ and reference values were, respectively, $122 \pm 92 \mathrm{UI} / \mathrm{L}, 6$ to $38 \mathrm{UI} / \mathrm{L}$, and $<35 \mathrm{UI} / \mathrm{L}$. Positive CMV-PCR in fetal blood was also defined when there was amplification of virus DNA in amniotic fluid or fetal blood sample by Enzymatic Activation / Lightcycle ${ }^{\circledR}$. Specific IgM (Konelab 30 Immunoturbidimetry ${ }^{\circledR}$ ) was considered positive titter when value was greater than $6 \mathrm{~g} / \mathrm{L}$.

\section{$\underline{\text { Statistical Analysis }}$}

Data were collected and organized at Statistical Package for Social Sciences (SPSS - version 15.0). Two groups of fetus/ newborns were considered for analysis:

a) Symptomatic: those with signs of CMV disease at ultrasound evaluation or anomalies at physical examination.

b) Asymptomatic: those without any ultrasound or clinical evidence of CMV disease.

Laboratory findings as thrombocytopenia or increase in liver enzymes could be present at symptomatic or asymptomatic fetus/infants samples because they are not proved to be associated to bad prognosis.

For mean comparison, t-test was performed and for proportion comparison $\chi^{2}$ of Pearson or Fisher test. Statistical significance was reached when $\mathrm{p} \leq 0.05$. Sensibility, specificity, positive predictive value (PPV) and negative predictive value (NPV) were also performed, considering a confidence interval (CI) of $95 \%$.

\section{Ethics Considerations}

In all cases the parents agreed to non-invasive and invasive prenatal diagnostic tests offered in the routine assistance for patients with CMV infection during pregnancy.The study was approved by ethical committee. Privacy was guaranteed and patients were identified by number (from one to 13). Only clinical staff and researchers were able to access informations from the patients.

Termination of pregnancy was requested by parents in one case of serious fetal disease and accepted by specialists authorized professionals of IPP, according to French law [14], considering the prognosis of a severe disease without treatment at the moment of diagnosis.

\section{Results}

Almost all the women (except for one) were diagnosed as having seroconversion at first trimester of pregnancy. Thus, correlation of gestational age and infant disease was not performed. Five of them had positive first viremia. Two persisted positive in second sample and one of them was considered negative only at the sixth sample. All fetus/infants were considered infected due to positive CMV-PCR, but five of them were symptomatic and eight asymptomatic.

Gestational age of amniocentesis ranged from 19 to 32 weeks of pregnancy with a mean of 24.6 weeks and a median of 23 weeks. Only two patients were submitted to the procedure after completed second trimester (at 31 and 32 weeks, respectively). Negative viremia was demanded to allow procedure.

Amniocentesis was performed in all women at least once. First viral load at amniotic fluid varied from 1,100 to 5,000,000 copies/mL. Mean value was 1,346,175 copies/mL and median was 860,000 copies $/ \mathrm{mL}$. There was no difference in values of mean viral load in amniotic fluid considering children with symptoms (1,560,375 copies/mL) and those without symptoms $(917,775$ copies $/ \mathrm{mL})$, presenting $\mathrm{p}=0.53$.

Mean gestational age in the first cordocentesis was 26.1 weeks and median was 26 weeks. Viral load, IgM, platelets, GGT, ASAT and ALAT values were also compared, considering symptomatic and asymptomatic infants. There were no statistical differences between groups, considering these parameters in fetal blood samples (Table 1).

Besides, samples of cordon at delivery were collected in twelve infants to perform viral load, because in one case termination of pregnancy was demanded by parents. There was no difference in viral load, platelets, GGT and ALAT when comparing infants with and without symptoms, but values of IgM and ASAT were increased in symptomatic ones, having $\mathrm{p}=0.03$ for both parameters (Table 1 ).

Analyses of platelet, GGT, ASAT and ALAT values as normal and abnormal and $\operatorname{IgM}$ as positive or negative were also performed, considering the presence or absence of symptoms in infants. None of these parameters presented statistical difference to predict symptoms, except ASAT of cordon samples at delivery $\left(\chi^{2}=5.6\right.$ and $\left.\mathrm{p}=0.02\right)$, as presented in Table 2.

Fetal involvement detected at ultrasound evaluations during pregnancy follow up did not show statistical difference when compared to IRM findings $\left(\chi^{2}=2.76\right.$ and $\left.p=0.1\right)$ or when considering symptomatic infants at birth $\left(\chi^{2}=0.12\right.$ and $\mathrm{p}=$ $0.73)$. Seven cases had from one to three lesions detected at antenatal ultrasound. IRM showed anomalies in only two cases (one had only one lesion and the other had two). Signs after birth were presented in five $(38.5 \%)$ infants, having one to seven anomalies. Anomalies at prenatal ultrasound, prenatal IRM and postnatal findings are presented at Table 3.

Sensibility, specificity, positive and negative predictive value of ultrasound anomalies to detect symptomatic infants were, respectively, 80\% (CI 95\%: 29.9-98.9), 62.5\% (CI 95\%: 
Table 1. Comparison of mean values of viral load, IgM, GGT, ASAT and ALAT values in cordocentesis and cordon samples at delivery of infants from women with CMV infection during pregnancy, considering absence or presence of symptoms (IPP, January 2005 to October 2006)

\begin{tabular}{|c|c|c|c|c|}
\hline & & Mean & Standard deviation (SD) & p value \\
\hline Cordocentesis & Symptoms & & & \\
\hline \multirow[t]{2}{*}{ Viral load (copies/mL) } & No & $19,884.4$ & $30,369.6$ & 0.98 \\
\hline & Yes & 20,240 & $21,969.8$ & \\
\hline Cordocentesis & Symptoms & & & \\
\hline \multirow[t]{2}{*}{$\operatorname{IgM}(g / d L)$} & No & 8.4 & 6.9 & 0.49 \\
\hline & Yes & 14.7 & 24.9 & \\
\hline Cordocentesis & Symptoms & & & \\
\hline \multirow[t]{2}{*}{ Platelets (cells/mm³) } & No & 183,500 & 47,395 & 0.90 \\
\hline & Yes & 179,500 & $53,394.8$ & \\
\hline Cordocentesis & Symptoms & & & \\
\hline \multirow[t]{2}{*}{ GGT(UI/L) } & No & 188.8 & 130.6 & 0.26 \\
\hline & Yes & 303.8 & 202.1 & \\
\hline Cordocentesis & Symptoms & & & \\
\hline \multirow[t]{2}{*}{ ASAT (UI/L) } & No & 20.8 & 2.8 & 0.15 \\
\hline & Yes & 28 & 10.8 & \\
\hline Cordocentesis & Symptoms & & & \\
\hline \multirow[t]{2}{*}{ ALAT (UI/L) } & No & 0 & 0 & 0.17 \\
\hline & Yes & 2.3 & 4 & \\
\hline Cordon sample - Delivery & Symptoms & & & \\
\hline \multirow[t]{2}{*}{ Viral load (copies/mL) } & No & $8,627.9$ & $21,774.2$ & 0.25 \\
\hline & Yes & 367,440 & $800,854.7$ & \\
\hline Cordon sample - & Symptoms & & & \\
\hline \multirow[t]{2}{*}{ Delivery $\operatorname{IgM}(\mathrm{g} / \mathrm{dL})$} & No & 8.8 & 9.6 & 0.03 \\
\hline & Yes & 46.4 & 25.4 & \\
\hline Cordon sample - Delivery & Symptoms & & & \\
\hline \multirow[t]{2}{*}{ Platelets $\left(\right.$ cells $/ \mathrm{mm}^{3}$ ) } & No & 255,800 & 66,213 & 0.20 \\
\hline & Yes & 183,000 & 87,719 & \\
\hline Cordon sample - Delivery & Symptoms & & & \\
\hline \multirow[t]{2}{*}{ GGT(UI/L) } & No & 141.3 & 59.9 & 0.28 \\
\hline & Yes & 302.4 & 268.6 & \\
\hline Cordon sample - Delivery & Symptoms & & & \\
\hline \multirow[t]{2}{*}{ ASAT (UI/L) } & No & 35 & 8.3 & 0.03 \\
\hline & Yes & 78 & 29.5 & \\
\hline Cordon sample - Delivery & Symptoms & & & \\
\hline \multirow[t]{2}{*}{$\operatorname{ALAT}(\mathrm{UI} / \mathrm{L})$} & No & 12.8 & 4.6 & 0.4 \\
\hline & Yes & 19.4 & 14.1 & \\
\hline
\end{tabular}

Table 2. Comparison between abnormal and normal values of platelets, GGT, ASAT and ALAT and positive or negative values of IgM from cordocentesis and cordon samples at delivery of infants from women with CMV infection during pregnancy, considering absence or presence of symptoms (January 2005 to October 2006)

\begin{tabular}{lcc}
\hline & $\boldsymbol{\chi 2}^{\mathbf{2}}$ & p value \\
\hline Cordocentesis - IgM & 0.17 & 0.68 \\
Cordocentesis - Platelets & 0.3 & 0.58 \\
Cordocentesis - GGT & 1.5 & 0.22 \\
Cordon sample - Delivery IgM & 2.7 & 0.1 \\
Cordon sample - Delivery Platelets & 0.03 & 0.86 \\
Cordon sample - Delivery GGT & 0.23 & 0.64 \\
Cordon sample - Delivery ASAT & 5.6 & 0.02 \\
\hline
\end{tabular}

*ASAT and ALAT in cordoncenteses and ALAT in cordon samples presented all normal values and statistics analysis was not performed. 
Table 3. Main anomalies identified by ultrasound, IRM and clinical evaluation after birth of fetus/infants of women with CMV seroconversion during pregnancy (IPP, January 2005 to October 2006)

\begin{tabular}{|c|c|c|c|}
\hline Case & Prenatal US & Prenatal IRM & Postnatal findings \\
\hline 1 & Wall hyperdensity of thalamic arteries & Normal & Asymptomatic \\
\hline \multirow[t]{7}{*}{2} & Intrauterine Growth Restriction & Normal & Anemia \\
\hline & Hepatosplenomegaly & & Neutropenia \\
\hline & & Thrombocytopenia & \\
\hline & & Cholestasis & \\
\hline & & Prematurity & \\
\hline & & Petechias & \\
\hline & & Subepemdimal cysts & \\
\hline \multirow[t]{5}{*}{3} & Intrauterine Growth Restriction & Normal & Prematurity \\
\hline & Periventricular calcifications & & Encephalitis \\
\hline & Intestinal hyperdensity & & Renal insufficiency \\
\hline & & Respiratory distress & \\
\hline & & Enteropathy & \\
\hline 4 & Normal & Normal & Trident elf \\
\hline 5 & Germinative zone cysts & Normal & Asymptomatic \\
\hline 6 & Subependimal cysts & Bilateral temporal cysts & Asymptomatic \\
\hline \multirow[t]{2}{*}{7} & Germinative zone cysts & Normal & Trident elf \\
\hline & Temporal cysts & & Germinative zone cysts \\
\hline 8 & Normal & Normal & Asymptomatic \\
\hline \multirow[t]{4}{*}{$9 *$} & Hypercogenicity of frontal horn of & Periventricular cysts & Hepatomegaly \\
\hline & lateral ventricules & Bilateral subependimal & Pericardial effusion \\
\hline & cysts & Pleural effusion & \\
\hline & Periventricular leucomalacy & & \\
\hline 10 & Normal & Normal & Asymptomatic \\
\hline 11 & Normal & Normal & Asymptomatic \\
\hline 12 & Normal & Normal & Asymptomatic \\
\hline 13 & Normal & Normal & Asymptomatic \\
\hline
\end{tabular}

* Termination of pregnancy.

25.9-89.9), 57.1\% (CI 95\%: 20.2-88.2), 83.3\% (CI 95\%:36.599.1).

Another clinical parameter evaluated was birth weight. Although there was a tendency of symptomatic infants presenting lower values (mean 2,515 g) comparing to asymptomatic ones $(3,274 \mathrm{~g})$, statistical difference was not observed $(\mathrm{p}=0.08)$. All children had also positive PCR in urine samples collected in the first three days of life, except for one not performed (termination of pregnancy).

\section{Discussion}

Although CMV vertical transmission occurs in about 30 to $57 \%$ of cases of seroconversion during pregnancy [1,5], all cases presented in this series were considered as infected due to positive PCR in amniotic fluids. This diagnostic method has being considered of greater sensitivity according to several authors and has been improving [6,7,15-18]. Correlation with fetus and infants exams was $100 \%$ once all studied cases had also positive PCR in cordocentesis, cord blood and urine samples just after birth (except for one case not performed in urine sample due to termination of pregnancy).
Values of viral load in amniotic fluid are being studied as prognostic markers of infant infection. No correlation was found in the present series, but Revello et al.[15] found higher DNA levels in amniotic fluid of mothers of symptomatc infants, although the difference was not statistically significant when compared to asymptomatic ones. Guerra et al. [9] reported that values greater than 1,000 copies $/ \mathrm{mL}$ were predictive of fetal infection and greater than 100,000 copies/mL were predictive of infant symptoms.

Considering maternal viremia, no correlation was observed between positivity of this parameter and infant symptoms $\left(\chi^{2}\right.$ $=0.18$ and $p=0.91)$. No anomaly was observed in the infant whose mother had persistent viremia (five samples), except for increase of GGT and ASAT in cordon samples at delivery. Brancart et al. [19] studied viral load in maternal blood of 35 CMV infected women and they also found no correlation to infant symptoms.

Although ultrasound findings during pregnancy also did not had statistical significance when considering symptomatic and asymptomatic infants at birth in the present series, some studies reported correlation with image anomalies and 
neurological disabilities. Ultrasound anomalies had a strong correlation with poor outcome for the neonate in Enders study [7]. Noyola et al. [10] reported that presence of microcephaly and abnormal signs at computed tomography were related to poor prognosis. Ruga at al. [12] followed CMV congenitally infected infants and six of seven children that presented cerebral anomalies in computed tomographic images had some neurological impairment during 53 months of follow up.

In the present series, five of 13 infants were considered symptomatic, corresponding to $38.5 \%$ of them, much higher than $10 \%$ reported $[1,5]$. Symptoms of systemic involvement were identified in three of them (Table 3) and two had only images at ultrasound follow up after birth. Greater rates of symptomatic infection was also reported in the study of Enders et al. [7] with 19 from 33 infected infants (57.6\%). Lanari et al. [11] has found 22 (37.9\%) symptomatic infants of $58 \mathrm{CMV}$ congenitally infected newborns. But these authors included among symptomatic infants those with laboratory anomalies as thrombocytopenia and increase of ALAT.

Isolated hematological and biochemistry anomalies were not considered to differ between symptomatic and asymptomatic infants. In 11 cases $(84,6 \%)$, at least one anomaly was present, considering platelets count or GGT or ASAT values at cordocentesis or cordon blood sample at delivery, even in the absence of clinical and image signs. ALAT was normal in all cases, considering reference values. This is also important to emphasize that ASAT presented statistical difference when comparing symptomatic and asymptomatic infants, considering mean values and also abnormal/normal parameters.

However, there is no evidence that these isolated parameters are related to severe prognosis. But, in a prospective study, Liesnard et al. [6] found that thrombocytopenia diagnosed at cordocentesis was associated with severe fetal disease and was presented in nine $(31 \%)$ infected infants. GGT was also increased in five (17.2\%); eight (27.6\%) had symptoms and only two $(6.9 \%)$ presented signs of systemic disease. Thus, hematological and biochemical values can be important parameters related to infected infants, guiding their follow up.

Although the present study did not show differences of mean viral load of fetal blood from cordocentesis or cordon samples at delivery when comparing symptomatic and asymptomatic infants, some authors found that CMV-PCR seems to be higher in symptomatic ones. Guerra et al. [9] reported that values greater than 1,000 copies/mL were predictive of fetal infection and greater than 100,000 copies/ $\mathrm{mL}$ were predictive of infant symptoms. Revello et al. [15] found higher DNA levels in amniotic fluid from mothers of symptomatic infants, although the difference was not statistically significant. In the study of Lanari et al. [11], viral load in infant blood higher than 10,000 copies/mL was related to sequels and DNA levels lower than 1,000 had a negative predictive value for sequels of $95 \%$.
In the present study, another variable correlated to infant symptoms was IgM antibodies mean levels of cordon samples $(\mathrm{p}=0.03$ - Table 1$)$, but positive or negative value was not related to presence of symptoms $(\mathrm{p}=0.1$ - Table 2$)$. Griffiths et al. [20] had already presented a study that correlated higher levels of IgM to symptomatic infants. In a prospective study of congenital infection from primary and recurrent maternal disease, Fowler [21] reported that, although presence of antibodies did not prevent transmission, fetus damage was less frequent in those cases with previous infection.

In the study of Revello et al. [23], the presence of IgM in newborn sample had sensibility of $70.7 \%$ to detect infant infection while presence of DNA had sensibility of $100 \%$. In this same study, level of IgM also was not predictive of symptoms $(p=0.44)$, while antigenemia $(p=0.005)$, viremia $(p$ $=0.009)$ and DNA level $(\mathrm{p}=0.018)$ were statistically correlated to infant disease. However, persistence of IgM antibodies was longer in symptomatic infants than those who were not symptomatic $(\mathrm{p}=0.045)$, as were also antigenemia, viremia and DNAnemia. In another publication, Revello et al. [23] found that all these parameters were predictive of infant infection in following order: DNAnemia $(82.3 \%), \operatorname{IgM}(57.9 \%)$, antigenemia $(57.9 \%)$, and viremia $(55.5 \%)$ and they concluded that combining virologic, laboratory and ultrasound evaluation can contribute to a better prognosis of fetal infection. The study of Enders at al. [7] showed sensitivity of IgM of $68.7 \%$ to diagnose symptomatic disease in infant and the authors also emphasized the importance of associating exams to improve prenatal diagnostic.

Considering birth weight, the study of Santos et al. [4] compared CMV infected and not infected newborns. There was no difference among groups $(\mathrm{p}=0.11)$. In the present study, comparison among symptomatic and asymptomatic infants was performed once all of them were infected and difference was not observed $(\mathrm{p}=0.08)$.

It is important to consider that it is possible to avoid vertical transmission or severity of disease in infants with maternal administration of specific immunoglobulin during pregnancy. Nigro et al. [24] compared two groups of CMV exposed fetus whose mothers received specific immunoglobulin or not. Prevention of vertical transmission was greater in the former group ( $\mathrm{p}=0.04)$. When considering infected fetus, immunoglobulin showed efficacy in reducing severity of disease $(\mathrm{p}<0.001)$.

Identification and follow up of infected fetus is also important to consider treatment. Gancivlovir has been studied for symptomatic infants in order to reduce sequels as chorioretinitis, thrombocytopenia and anemia. Greater benefits are proved to be related to hearing improvement, although doses and duration of treatment are not already defined [25-30].

The present study included just a few patients to discuss external validity. However, results pointed out the importance of extensive evaluation of infected women at their infants, as during prenatal diagnosis as in postnatal follow up in order to allow prevention and treatment of vertical disease. 


\section{References}

1. Revello M.G., Gerna G. Diagnosis and management of human cytomegalovirus infection in the mother, fetus and newborn infant. Clin Microbiol Rev 2002;15(4):680-715.

2. Collinet P., Subtil D., Houfflin-Debarge V., et al. Routine CMV screening during pregnancy. Eur J Obstet Gynecol Reprod Biol 2004; $114 ; 3-11$.

3. Yamamoto A.Y., Mussi-Pinhata M.M., Cristina P., et al. Congenital cytomegalovirus infection in preterm and full-term newborn infants from a population with a high seroprevalence rate. Pediatr Infect Dis J 2001 Feb;20(2):188-92.

4. Santos D.V., Souza M.M., Gonçalves S.H., et al. Congenital cytomegalovirus infection in a neonatal intensive care unit in brazil evaluated by PCR and association with perinatal aspects. Rev Inst Med Trop Sao Paulo 2000;42(3):129-32.

5. Audibert F., Goffinet F. Pour ou contre le depistage systématique du CMV chez la femme inceint? Gynécologie Obstétrique Fertilité 2002;30:994-1004.

6. Liesnard C., Donner C., Bracart F., et al. Prenatal diagnosis of congenital cytomegalovirus infection: prospective study of 237 pregnancies at risk. Obstet Gynecol. 2000 Jun;95(6 Pt 1):881-8.

7. Enders G., Bader U., Lindemann L., et al. Prenatal diagnosis of congenital cytomegalovirus infection in 189 pregnancies with known outcome. Prenat Diagn 2001;21:362-77.

8. Revello M.G., Zavattoni M., Furione M., et al. Quantification of Human Cytomegalovirus DNA in amniotic fluid of mothers of congenitally infected fetus. J Clin Microbiol 1999;37(10):3350-1.

9. Guerra B., Lazzarotto T., Quarta S., et al. Prenatal diagnosis of symptomatic congenital cytomegalovirus infection. Am J Obstet Gynecol 2000; $183: 476-82$.

10. Noyola D.E., Demmler G.J., Nelson C.T., et al. Houston Congenital CMV Longitudinal Study Group. Early predictors of neurodevelopmental outcome in symptomatic congenital citomegalovirus infection. J Pediatr 2001;138:325-31.

11. Lanari M., Lazarotto T., Venturi V. Neonatal Cytomegalovirus blood load and risk of sequelae in symptomatic and asymptomatic congenitally infected newborns. Pediatrics 2006;117(1):e76-83.

12. Ruga E.M., Balao I., Manara R., et al. Neuroimaging fidings and neurological outcome in symptomatic congenital cytomegalovirus infection. Poster (C40) - Prognostic markers and counselling. Conference on Congenital Cytomegalovirus Infection. November 5-7, 2006 - Orvieto-Italy.

13. Lipitz S., Yagel S., Shalev E., et al. Prenatal Diagnosis of fetal primary cytomegalovirus infection. Obstet Gynecol 1997;89:763-7.

14. Journal Officiel de la Republique Francaise. 18 janvier 1975, page 739. Loi 75-17 de 17/01/1975.

15. Revello M.G., Sarasini A., Zavattoni M., et al. Improved Prenatal diagnosis of congenital human cytomegalovirus infection by a modified nested polimerase chain reaction. J Med Virol 1998;56:99-103.

16. Lazzarotto T., Guerra B., Spezzacatena P., et al. Prenatal diagnosis of Congenital Cytomegalovirus Infection. J Clin Virol 1998;36(12):3540-4.
17. Bodeuse M., Hubinont C., Bernard P., et al. Prenatal diagnosis of human cytomegalovirus by culture and polimerase chain reaction: 98 pregnancies leading to congenital infection. Prenat Diagn 1999; 19:314-7.

18. Lipitz S., Achiron R., Zalel Y., et al. Outcome of pregnancies with vertical transmission of primary cytomegalovirus infection. Obstet and Gynecol 2002;100(3):428-33.

19. Brancart F., Donner C., Vincart B., et al. Are HCMV DNA loads in urine and blood of infected pregnant women prognostic markers of congenital infection in their newborns? Oral Communication C-26 Prognostic markers and counselling. Conference on Congenitatal Cytomegalovirus Infection. Ovieto-Italy. 5-7 November $\mathbf{2 0 0 6 .}$

20. Griffiths P.D., Stagno S., Pass R.F., et al. Congenital cytomegalovirus infection: diagnostic and prognostic significance of the detection of specific immunoglobulin $\mathrm{M}$ antibodies in cord serum. Pediatrics 1982;69(5):544-9.

21. Fowler K.B., Stagno S., Pass R.F., et al. The outcome of congenital cytomegalovirus infection in relation to maternal antibody status. N Engl J Med 1992 Mar 5;326(10):663-7.

22. Revello M.G., Zavattoni M., Baldanti F., et al. Diagnostic and prognostic value of human cytomegalovirus load and $\operatorname{IgM}$ antibody in blood of congenitally infected babies. J Clin Virol 1999; $14: 57-66$.

23. Revello M.G., Zavattoni M., Sarasini A., et al. Prenatal diagnostic and prognostic value of human cytomegalovirus load and $\operatorname{IgM}$ antibody response in blood of congenitally infected fetus. J Infect Dis 1999; 180:1320-3.

24. Nigro G., Adler S.P., La Torre R., Best ${ }^{a}$ M. Congenital Cytomegalovirus Collaboration Group. Passive immunization during pregnancy for congenital cytomegalovirus infection. $\mathrm{N}$ Engl J Med 2005;353(13):1350-62.

25. Nigro G., Scholz H., Bartmann U. Ganciclovir therapy for symptomatic congenital cytomegalovirus infection in infants: a two regimen experience. J Pediatr 1994;124(2):318-22.

26. Whitley R.J., Cloud G., Gruber W., et al. Ganciclovir treatment of symptomatic congenital cytomegalovirus infecion: results of a phase II study. National Institute of Allergy and Infectious Diseases. J Infect Dis 1997;175(5):1080-6.

27. Kimberlin D.W., Lin C.Y., Sanchez P.J., et al. National Institute of Allergy and Infectious Diseases Collaborative Antiviral Study Group. Effect of ganciclovir therapy on hearing in symptomatic congenital cytomegalovirus disease involving the central nervous system: a randomized, controlled trial. J Pediatr $\mathbf{2 0 0 3}$ Jul;143(1):16-25.

28. Michaels M.G., Greenberg D.P., Sabo D.L., Wald E.R. Treatment of children with congenital cytomegalovirus infection with ganciclovir. Pediatr Infect Dis J 2003;22(6):504-9.

29. Tanaka-Kitajima N.T., Sugaya N., Futatani T., et al. Ganciclovir therapy for congenital cytomegalovirus infection. Pediatr Infect Dis J 2005;24:782-5.

30. Adler S.P., Nigro G., Pereira L. Recent advances in the prevention and treatment of congenital cytomegalovirus infections. Semin Perinat 2007 Feb;31(1):10-8. 